David Lewis's Humean Theory of Objective Chance

Author(s): Barry Loewer

Source: Philosophy of Science, Vol. 71, No. 5, Proceedings of the 2002 Biennial Meeting of The Philosophy of Science Association $\langle$ break $\rangle /$ break $>$ Part II: Symposia

Papers $\langle$ break $></$ break $>$ Edited by Sandra D. Mitchell (December 2004), pp. 1115-1125

Published by: The University of Chicago Press on behalf of the Philosophy of Science Association

Stable URL: http://www.jstor.org/stable/10.1086/428015

Accessed: 10/02/2011 23:03

Your use of the JSTOR archive indicates your acceptance of JSTOR's Terms and Conditions of Use, available at http://www.jstor.org/page/info/about/policies/terms.jsp. JSTOR's Terms and Conditions of Use provides, in part, that unless you have obtained prior permission, you may not download an entire issue of a journal or multiple copies of articles, and you may use content in the JSTOR archive only for your personal, non-commercial use.

Please contact the publisher regarding any further use of this work. Publisher contact information may be obtained at http://www.jstor.org/action/showPublisher?publisherCode=ucpress.

Each copy of any part of a JSTOR transmission must contain the same copyright notice that appears on the screen or printed page of such transmission.

JSTOR is a not-for-profit service that helps scholars, researchers, and students discover, use, and build upon a wide range of content in a trusted digital archive. We use information technology and tools to increase productivity and facilitate new forms of scholarship. For more information about JSTOR, please contact support@jstor.org. 


\title{
David Lewis's Humean Theory of Objective Chance
}

\author{
Barry Loewer $\$$
}

\begin{abstract}
The most important theories in fundamental physics, quantum mechanics and statistical mechanics, posit objective probabilities or chances. As important as chance is there is little agreement about what it is. The usual "interpretations of probability" give very different accounts of chance and there is disagreement concerning which, if any, is capable of accounting for its role in physics. David Lewis has contributed enormously to improving this situation. In his classic paper "A Subjectivist's Guide to Objective Chance" he described a framework for representing single case objective chances, showed how they are connected to subjective credences, and sketched a novel account what they are within his Humean account of scientific laws. Here I will describe these contributions and add a little to them.
\end{abstract}

In David Lewis's framework, described in his paper "A Subjectivist's Guide to Objective Chance" (1986) chances are given by propositions in the language of fundamental physics that specify that a type of event will (or won't) occur at a specific time (or during a particular time interval) in a specific location (or region)). Such chances are "single case" since they apply to token fundamental events. The chance of an event $A$ occurring at time $t$ may itself change during the times prior to $t$ so chances are time indexed. At $t$ the chance of $A$ occurring at $t+2$ may be $\frac{1}{2}$. But at $t+1$ the chance of $A$ occurring at $t+2$ may increase to .9. At $t$ the chance of every proposition about times entirely prior to $t$ is either 0 or 1. If we think of time as discrete then the assignment of chances gives rise to a tree structure branching toward the future. At each node the branches are the possible futures from that node each weighted by its chance from the time of that node. If at each node there is a chance (or

$\dagger$ To contact the author, please write to: Department of Philosophy, Rutgers University, Davison Hall 135, New Brunswick, NJ 08901; e-mail: loewer@rci.rutgers.edu.

$\$$ Thanks to David Albert, Tim Maudlin, Frank Arntzenius, and David Papineau for comments on various versions of this paper.

Philosophy of Science, 71 (December 2004) pp. 1115-1125. 0031-8248/2004/7105-0041\$10.00

Copyright 2004 by the Philosophy of Science Association. All rights reserved. 
chance density) for every possible subsequent state the chances of each possible future (sequence of states) is determined.

In Lewis's framework all chances fall under what he calls "history to chance conditionals." These are laws of the form "if $h$ is the actual history up to and including $t$ the chance at $t$ of $S$ at $t^{\prime}\left(t^{\prime}>t\right)$ is $x .{ }^{\prime 1}$ In all the candidates for fundamental theories I know the dynamical chances at $t$ are determined by the state at $t$. So the history to chance laws are state to chance conditionals of the form "if the state at $t$ is $S^{*}$ then the chance at $t$ of the state at $t^{\prime}$ being $S$ is $x$." The chance at $t$ of a proposition $R$ is the sum of the chances of the possible histories branching from the actual history at $t$ at which $R$ is true.

Chances are involved in the causal nexus. Chancy events are caused by events that raise their chances and chances are involved in explaining the events they are chances of. It is difficult to see how chances can play their roles in laws, explanation, and causation unless they are objective; i.e. their existence and values are independent of our beliefs. But objective chance is related to belief in a special way; chances rationally guide belief. It is not just that our beliefs ought to track truths about chance (as they ought track truths about various other matters) but that beliefs about the chance of an event rationally constrain beliefs about that event itself. Lewis proposes a principle which he thinks captures how chance should guide belief called "the Principal Principle" (PP). The (PP) says, roughly, that a person $M$ 's degree of belief (at $t$ ) that $A$ conditional on the proposition that the chance of $A$ (at $t$ ) is $x$ should be equal to $x$; with the qualification that at $t$ she has no information about $A$ that is inadmissible. Information about $A$ is inadmissible if it is information about $A$ over and above information about $A$ 's chance.

PP: $C(A / P(A)=x \& T)=x$ (where $T$ is any information admissible wrt $A$ ).

So, for example, if you believe that the coin in front of you has a chance of $\frac{1}{2}$ of landing heads when flipped then your degree of belief conditional on that and any other information that doesn't tell you anything about the outcome of the flip (except what its chance is) should also be $\frac{1}{2}$. While Lewis initially glosses admissibility as above he later suggests that any

1. Lewis says that the conditionals are stronger than material conditionals. If the chances are fundamental then it follows from Lewis's account of chances and laws hat these conditionals are nomologically necessary. 
information about times prior to the time of $A$ and any laws of natureincluding laws that specify chances - are always admissible relative to $A{ }^{2}$

The PP enables one to infer credences about frequencies from credences about chance. So, for example if you think that the chance of an event of type $A$ in circumstances of type $E$ is $x$ and repetitions of $E$ are independent then the PP implies that if there are many repetitions of $E$ your degree of belief that the frequency with which $A$ events occur in $E$ situations is approximately $x$ should be close to 1 . Lewis shows how PP fits into Bayesian inference to yield an epistemology for chance propositions. If $H$ and $H^{*}$ are exclusive and exhaustive hypotheses that specify chances for experimental outcomes then the PP says that one's conditional credences $\left(C(e / H), C\left(e / H^{*}\right)\right)$ should be the chances $H$ and $H^{*}$ respectively assign to $e$. Given prior credences for $H$ and $H^{*}$ Bayes theorem yields the posterior credences $C(H / e)$ and $C\left(H^{*} / e\right)$.

We have seen that chances are janus-faced. On the one hand they are objective features of reality involved in laws and causation and, on the other hand, they rationally constrain degrees of beliefs via the (PP). We would like an account that explains both of these aspects and shows us how one property can possess both kinds of features. That is, how an objective feature of a situation $S$ (the chance of $S$ resulting in event $e$ ) which is part of the causal order and is metaphysically distinct from $e$ (it is possible for it to occur without $e$ ) rationally constrain what degree of belief we should have in e's occurring? That something can possess both features can appear very puzzling; as puzzling as the Mackie found ethical facts that allegedly are part of the causal order and also normatively constrain what we should value. ${ }^{3}$

Lewis's framework seems designed for so called "propensity chances."4 But toward the end of the paper he suggests a novel account which is quite different from standard propensity accounts. In a longer version of this paper I survey the standard interpretations of probability - actual and modal frequency accounts, propensity accounts of various sorts, and subjectivist accounts - with an eye to evaluating whether any yields an a

2. What is admissible for a given person will in general depend on her credence function. Lewis can be understood as suggesting that rational credence functions are ones in which information about the past and about laws are relevant to a proposition $A$ only by being relevant to the chance of $A$.

3. This analogy between chances and values is also made by Black (1998) who thinks it poses a problem for propensity accounts of chance and at least as great a problem for Lewis's account of chance that I discuss later. As the reader will soon see I agree with the first but not the second of these claims.

4. On the propensity account the chance at t of situation $S$ result in $E$ is a measure of the degree of the disposition (or propensity) of $S$ at $t$ to bring about $E$. 
notion of chance capable of playing its role in physical theories and also as guiding belief via the PP. My conclusion is that none of the usual suspects measure up. Those which interpret chance as an objective feature of fundamental events may account for its role in physics but make it utterly mysterious why chances so construed should guide our beliefs in conformity with the PP. Subjectivist accounts have no trouble with PP but are not credible as accounts of the chances that occur in physics. This opens the field for Lewis's novel account.

Lewis's account of chance is part of his more general Humean account of laws including laws of chance; the Best System Account (BSA). The account is "Humean" in that it characterizes laws as certain regularities and which regularities are laws is determined by the total pattern of instantiation of categorical properties. ${ }^{5}$ Here is how the BSA works. Let $L$ be a language whose atomic predicates express only fundamental properties (Lewis calls these "perfectly natural properties"), spatio-temporal predicates (e.g. distances among points) and mathematics/logic. The truths of $L$ specify the geometrical structure of space-time and the fundamental properties that are instantiated at each point. Lewis thinks that it is the job of fundamental physics to inventory these properties and suggests that they are or are similar to quantities like mass, charge, electromagnetic field values and so forth. All truths supervene on the totality of truths expressible in $L$ (that is what makes $L$ fundamental). A deductive system in $L$ is a set of sentences of $L$. The laws are defined as follows:

Take all deductive systems whose theorems are true. Some are simpler better systematized than others. Some are stronger, more informative than others. These virtues compete: An uninformative system can be very simple, an unsystematized compendium of miscellaneous information can be very informative. The best system is the one that strikes as good a balance as truth will allow between simplicity and strength. How good a balance that is will depend on how kind nature is. A regularity is a law IFF it is a (contingent) theorem of the best system. (1994, 478)

There may be further conditions that should be put on the law $g$ iving systems. For example, that it counts against a system if it is compatible with events that are not covered by its laws.

Chances enter the picture by letting deductive systems include sentences that specify the chances of events.

5. A property are categorical just in case its involvement in laws is not essential to it. Lewis characterizes "Humean Supervenience" as the doctrine that (i) all the fundamental natural properties instantiated in the world are categorical and (ii) all truths supervene on the pattern of instantiation of fundamental properties. 
Consider deductive systems that pertain not only to what happens in history, but also to what the chances are of various outcomes in various situations - for instance the decay probabilities for atoms of various isotopes. Require these systems to be true in what they say about history. . . . Require also that these systems aren't in the business of guessing the outcomes of what, by their own lights, are chance events; they never say that A without also saying that A never had any chance of not coming about. $(1994,480)$

The axiom systems that vie for the title "Best" are supplemented with a so far un-interpreted function $P t$ that is thought of as assigning chances to certain propositions at times. All candidates for Best system must be true. The virtues that make for a good system are simplicity, strength, and fit. Simplicity is measure in terms of order of differential equations, number of parameters, length of the conjunction of axioms etc. Strength is measured in terms of informativeness (possibilities characterized in terms of natural predicates excluded). Lewis suggests evaluating "fit" in terms of the likelihood of truths. The higher the chance a system assigns to the true history (or to segments of it given part of the history) the better its fit. So understood fit is a kind of informativeness appropriate for chance. The better a theory fits the facts the more it says about those facts. I will later discuss just how a chance theory provides information. For now let us just observe that these various virtues will typically trade off. Strength and fit can often be improved at the cost of simplicity and visa versa. By assigning chances systems sacrifice strength for fit but may also make great gains in simplicity. Lewis's account assumes that there is a set of fundamental natural properties and that there is a unique Best System and that it is both pretty simple and pretty informative. ${ }^{6}$ Assuming he is right about this the BSA will deliver a determinate collection of true generalizations. Let's call the contingent ones the " $L$-laws" and the chances entailed by the $L$-laws " $L$-chances."

Lewis's BSA is a big improvement over previous Humean accounts. Among its improvements are that it connects $L$-laws and $L$-chances with the criteria (simplicity, informativeness, and so forth) physicists actually employ. Further, it is able to distinguish lawful from accidental regularities and allow for vacuous laws. $L$-chances apply both to token events (the chance that this photon will pass through a that polarizer in the next second) and to type chances (the chance that a photon with polarization

6. Lewis suggests that if there is a tie then the laws are the generalizations common to both and that given the enormity of our world makes it unlikely that there are ties. If there is no very good systematization then the Humean should say that there are no laws. 
of type $X$ will pass through a polarizer of type $Y$ ). Also, while $L$-chances are connected to actual frequencies they can diverge arbitrarily from them.

Despite its attractions Lewis's account is not lacking in critics. The main worry is that Humean regularities - even the special ones given by the Best System of the world - are two weak to do the work of real laws. It has been claimed that Humean regularities (and specifically $L$-laws) are too anemic to support counterfactuals, provide explanations, be inductively confirmed as so forth. All of these objections have been adequately rebutted by Lewis and others. ${ }^{7}$ Even so it is not difficult to produce thought experiments that provoke non-Humean intuitions. ${ }^{8} \mathrm{I}$ think that the most effective reply for the Humean is to grant the point but argue that $L$-laws can play the role of laws and that non-Humean laws are metaphysically and epistemologically problematic. The main non-Humean competitor to Lewis's account claims that laws and chances involve facts over and above the history of the distribution of fundamental properties that in some way govern or guide the evolution of events. Call these $M$-laws. The trouble with $M$-laws is that it is hard to understand what the metaphor of governing comes to. How can the $M$-law, which is one thing, govern or produce other events? It is especially problematic when the fundamental laws involve propensity chances. How does a propensity guide the development of subsequent events? And, even more puzzling, why should, degrees of propensity guide rational belief? I think that critics of Lewis's account have no adequate answers to these questions. If there were no alternatives to positing - laws and chances we might have to accept ("with natural piety" as Armstrong puts it) the existence of $M$-law and propensities. But Lewis's account is a less metaphysical alternative and also, as we will see, has some very interesting further advantages over its metaphysical rivals.

But first, I need to discuss what Lewis called "a big bad bug" in his account of chance. The problem is that the Best Theory of a world may assign a non-zero $L$-chance at time $t$ to a possible future $F(t)$ which is logically incompatible with that $L$-chance. He calls such a future "undermining" since it undermines the theory that assigns it a positive chance. For example, suppose that world $w$ consists entirely of a sequence of 1000 "coin flips" occurring at discrete times about half of which are $h$ and half $t$ so that the Best Theory BT( $w$ ) for $w$ says that the flips form a Bernoulli

7. Objections along these lines are made by Armstrong, Dretske, Foster, among others. Lewis (1983) and Loewer (1996) contain responses.

8. These thought experiments involve describing situations in which it appears that the occurrent facts (and so regularities) are held fixed while the laws and chances vary. See for example Carroll (1994) and Tooley (1977). I discuss these and explain why they don't tell much against Lewis's account in my (1996). 
sequences with equal chances of $h$ and $t$ on each flip. Suppose the history to time $500 H(500)$ consists of an equal number of $h s$ and $t s$. At time 500 a future $F(500)$ consisting of 500 heads has, according to $\mathrm{BT}(w)$, a chance of $\frac{1}{2}$ to the 500 power. $F(500)$ is an undermining future relative to $\mathrm{BT}(w)$. By the PP $C(F(500) / H(500) \mathrm{BT}(w))=\frac{1}{2}$ to the 500th power. Let $w^{\prime}$ be the world composed of $H(500)+F(500)$. BT $\left(w^{\prime}\right)$ is certainly different from BT $(w)$. So BT $(w)$ is (supposedly) logically incompatible with $w^{\prime}$. But then $C(F(500) / H(500) \mathrm{BT}(w))=0$. Since this is correct the PP is false for $L$ chance. ${ }^{9}$ One can avoid an outright contradiction by claiming that $\mathrm{BT}(w)$ is not admissible. This is reasonable since $\mathrm{BT}(w)$ provides information about the future. But this is of little comfort since in general $\mathrm{BT}(w)$ provides information about the future. If it is never admissible than PP will be useless. Lewis observed that the problem is not specific to the BSA account but will arise on any account of chance compatible with Humean Supervenience on which chances are contingent. Since Lewis thought that the PP encapsulates "all we know about chance" this supposed incompatibility caused him to despair that his BSA could be defended. ${ }^{10}$

Lewis eventually proposed to solve the problem by replacing the PP with

NP: $C(A / T)=P(A / T)$.

Since $P(F(500) / \mathrm{BT}(w))=0$ the problem (as given in the example but the point is general) doesn't arise. The NP give almost the same results as the PP for $L$-chances and exactly the same results on any account of chance on which the true chance theory has chance $=1 .{ }^{11}$ Lewis seems content to think that by adopting the NP the BSA is saved. The fact that $L$-chances almost satisfy the NP (since the NP and the PP are quite close) allows us to count $L$-chances as genuine chances, at least as long as there is nothing that plays the chance role any better. A defense of that claim would of course involve showing that propensity chances don't exist.

Let's return to the question of why we should accept the PP or the NP? Lewis says, somewhat mysteriously, "I can see, dimly but well enough,

9. As we will see this argument is fallacious. One also needs to add to FH the statement that FH is the entire history of the world.

10. Propensity accounts of chance don't conflict with PP since chances are logically compatible with any histories. The very feature of propensity accounts that makes them metaphysically and epistemologically suspect (that worlds can completely agree with respect to their non-chance facts but differ in their chances and disagree enormously yet agree on their chances) is what allows them to conform to the PP.

11. On Lewis's account a chance theory will assign itself a chance less than 1. A propensity account typically will not assign chances to chance statements but it is compatible with those accounts to extend the chance distribution so that the chance theory obtains chance $=1$. 
how knowledge of frequencies and symmetries and best systems could constrain rational credence, ..." (Lewis 1994, 484). He seems to be suggesting that on the BSA account chances involve symmetries and frequencies and that these constrain rational degrees of belief. Unlike propensities but like actual frequencies $L$-chances supervene on non-chance propositions so a principle of rationality that constrains rational degrees of belief with respect to non-chance propositions can entail that chance propositions rational constrain belief. But it is not clear how the principle of indifference can support the PP with respect to $L$-chance (in fact it seems to conflict with it) and, in any case, the principle of indifference has little to recommend it. ${ }^{12}$ But I think that we can see how $L$-chances can constrain degrees of belief if we recall how they are characterized in terms of Best Theory. Recall that a Best System is one that best combines simplicity, informativeness, and fit. The fit of a theory is measured by the probability that it assigns to true propositions. Fit can be understood as a kind of informativeness - the information that probabilistic propositions provide concerning the propositions they attribute probability to. The higher the probability assigned to true propositions that more informative the theory (the higher the probability to false propositions the less informative and the more misleading the theory). But these probabilities are informative only to someone who is willing to let them constrain her degrees of belief. Now, suppose that someone decides to let the PP constrain her degrees of belief. Then for her the Best theory will be one that best combines simplicity and informativeness - including informativeness as evaluated in terms of the degrees of belief she assigns to true propositions. On this proposal the PP is "built into" the account of $L$-chance. It can constrain belief because that is part of the account of how a theory earns its title as "Best System."

Suppose that instead of adopting the PP someone decides to set her degrees of belief by a different principle; say the anti-PP; $C(A)=1-$ $P(A)$. When this principle is used to evaluate the informativeness of theories we will count a theory as more informative the lower the probability it assigns to true proposition. Of course the system that will be counted as Best when using this principle will differ from the Best theory characterized using the PP and the probabilities it defines then will differ (systematically) from the $L$-chances. Let's call them anti- $L$-chances. Anti$L$-chances exist just as much as $L$-chances. The important point is that someone who sets her degrees of belief by the anti-PP applied to anti$L$-chances will have degrees of belief that are exactly the same as someone who uses the PP as applied to $L$-chances. And, of course, there are count- 
less other principles that characterize alternative chance like notions ("chance like" because the characterize functions that satisfy the probability calculus) but which end the end recommend more or less the same degrees of belief (when applied to the chance notion they help characterize) as the PP does for $L$-chance. Perhaps we could use one of these other principles and one of these other notions of chance. The PP (and $L$-chance) recommends itself because it is the simplest.

The preceding considerations shows that a Humean who values possessing the Best Theory of the world (because that theory specifies by his Humean lights the nomological structure of the world) and whose Best Theory contains probability statements must adopt some principle that extracts information from information about the Humean mosaic from these statements. The PP is a way of doing that. In the process it provides an interpretation (i.e., truth conditions) for probability statements. So a Humean who values possessing the Best Theory has a reason to adopt the PP (or its correction the NP). It is worth noting what these considerations don't show. They don't establish that degrees of belief that fail to conform to the PP for $L$-chances are incoherent (i.e. violate the probability calculus). There are coherent credence functions that violate the PP for $L$-chance. Nor do they show that violation of the PP is a violation of some version of the principle of indifference. On natural construals the principle of indifference is incompatible with the PP. And of course we haven't shown that failure to set one's degrees of belief by the PP will lead to failure in one's decisions (say gambles on the outcomes of quantum experiments) in either the short or the long run. The most that could ever by shown is that failure would be probable but that is only significant given the PP. However, someone who violates the PP is, in a fairly straightforward sense, being irrational. On the one hand she accepts that a theory $T$ provides the best combination of simplicity and informativeness and that $T$ recommends that she have a degree of belief of $p$ in a proposition $A$ (as long as she has no inadmissible information wrt $A$ ) and yet she opts for a different degree of belief. Such a person is in the position of accepting that a certain person is the best source on what degrees of belief to have regarding certain matters and then opting for different degrees of belief. Notice that this rationale for the PP is not available for propensity chances. Without replying on the PP there is no non-question begging reason to think that setting one's degrees of belief by propensity chances will result in having high degrees of belief in truths and low degrees of belief in falsehoods. And since propositions about propensity chances are facts logically completely distinct from the propositions they assign chances to it is utterly mysterious why they should tell us anything about what degrees of belief to have in those propositions.

There are important theories in physics that assign chances to events 
even though the dynamical laws that occur in these theories are thoroughly deterministic. Two examples are statistical mechanics and Bohmian mechanics. Both contain principles or laws that specify probability distributions over initial conditions. There is a tradition of attempting to interpret these probabilities subjectively, as degrees of belief. But, for pretty much the reasons mentioned in our earlier discussion of subjective probabilities it is hard to see how subjective probabilities can underwrite the use of probabilities in explanation and laws. For example, within Bohmian mechanics the prohibition on super-luminal signaling follows from the specific Bohmian probability assumption over initial conditions. Other probability distributions permit super-luminal signaling. The failure of super-luminal signaling seems about as lawful as any generalization. But it would be awkward at best to maintain that its status as a law is due to our degrees of belief - to our ignorance!

It is not difficult to extend $L$-chances to cover initial condition probability distributions. If by adding such a proposition to a theory one makes a great gain in informativeness with little cost in simplicity than that probability distribution has earned its status as a law and the chances it specifies are as objective as dynamical $L$-chances. Arguably this is just the case with respect to the micro-canonical distribution in statistical mechanics and the Bohmian probability distribution within Bohmian mechanics. By adding the micro-canonical distribution to Newtonian laws the resulting system (and the proposition that the entropy in the distant past was much lower than currently) entails all of statistical mechanics. By adding the quantum equilibrium distribution $\left(P(x)=\Psi^{2}\right)$ to the Bohmian dynamical laws the resulting system entails standard quantum mechanics. In both cases enormous information gain is achieved with very little cost in simplicity. ${ }^{13}$

Even believers in propensity chances and metaphysical laws should grant (assuming that simplicity and informativeness are clear) that $L$ chances and $L$-laws exist. ${ }^{14}$ The issue between them and advocates of the BSA account is whether L-laws and chances are the chances and laws that are posited by fundamental theories like GRW and Bohmian Mechanics? Whether or not $L$-chances are the subject of these theories depends in part on whether $L$-laws are good candidates for being fundamental laws. If scientific laws can be identified with $L$-laws then the

13. See my "Determinism and Chance" (2001) for a development and defense of the idea that $L$-chances are compatible with determinism.

14. An interesting question is whether metaphysical laws and propensity chances will match or are likely to match the $L$ versions. It is hard to see how to argue that it is likely that they will match without appealing to the PP but, as I have argued, it is hard to see what justifies the PP for propensity chances. 
$L$-chances they may specify do, I think, play the role of chances in science. They are objective, governed by laws ( $L$-laws), enter into explanations, and underwrite a rationale for the PP. Of course this is a big "IF." The main competition for $L$-laws and $L$-chances are from more metaphysical views about laws and chance. It must be admitted that these metaphysical accounts fit some of intuitions concerning law and chance better than the $L$-versions do. In particular the intuition that very different systems of laws and chances can give rise to the same total history of occurrent events is quite strong. If the requirement that anything worthy of the names "law" and "chance" satisfy this intuition then HS accounts are dead in the water. But until it can be shown that this requirement is essential if laws and chances are to play the roles that science requires of them then I think we have little reason to give such deference to the intuition. And that is especially so for those who, like myself, find metaphysical laws and chances that are at once metaphysically independent of events and yet govern and their evolution and rationally guide our beliefs utterly mysterious.

\section{REFERENCES}

$\rightarrow$ Black, Robert (1998), "Chance, Credence and the Principal Principle", British Journal for the Philosophy of Science 49: 371-385.

Carroll, John (1994), Laws of Nature. Cambridge: Cambridge University Press.

$\rightarrow$ Lewis, David (1983), "New Work for a Theory of Universals", Australian Journal of Philosophy 61: 343-377.

- (1986), "A Subjectivist's Guide to Objective Chance", in Philosophical Papers, vol. 2. Oxford: Oxford University Press, 83-132.

$\rightarrow \longrightarrow$ (1994), "Chance and Credence: Humean Supervenience Debugged", Mind 103: 473 490.

Loewer, Barry (1996), "Humean Supervenience”, Philosophical Topics 24: 101-127.

$\rightarrow-(2001)$, "Determinism and Chance", Studies in History and Philosophy of Modern Physics 32: 609-620.

Tooley, Michael (1977), “The Nature of Laws”, Canadian Journal of Philosophy 7: 667-698. 